\title{
Influence of Age on Cardio-Respiratory Interaction Assessed by Joint Symbolic Dynamics
}

\author{
Muammar M. Kabir ${ }^{\# 1}$, Derek Abbott ${ }^{\# 2}$, Mathias Baumert \#3 \\ \# Centre for Heart Rhythm Disorders, School of Electrical and Electronics Engineering, The University of Adelaide \\ Adelaide, SA 5005, Australia \\ 1 muammar.kabir@adelaide.edu.au \\ 2 dabbott@eleceng.adelaide.edu.au \\ 3 mathias.baumert@adelaide.edu.au
}

\begin{abstract}
Cardio-respiratory dynamics exhibit complex behaviour and underlying mechanisms responsible for their interaction have not been fully understood. The aim of this paper was to validate the approach for detection of cardio-respiratory interaction based on joint symbolic dynamics using two simulated non-linearly coupled systems, and to investigate the influence of age on the interaction between cardiac and respiratory cycles in healthy subjects. For symbolic analysis, the time series were transformed into ternary symbol vectors based on the changes between two successive time points of each series. Subsequently, words of length ' 3 ' were formed and the correspondence between words of the two series was determined. The process was repeated using surrogate data to determine the effectiveness of the joint symbolic dynamics approach. Subsequently, the electrocardiograms (ECG) and respiratory signals of 20 young (age: 21-34 yrs) and 20 elderly (age: $68-85$ yrs) healthy subjects were collected from Physionet, from which the $R-R$ intervals and respiratory phases were extracted, respectively. Using the joint symbolic dynamics approach and an additional adaptive delay in the cardiac oscillator based on the maximization of the cross-correlation between the phases of respiration and the delayed R-R intervals, we found a significantly higher percentage of similarity in the symbolic dynamics of $R-R$ intervals and respiratory phases in young subjects compared to elderly subjects (22.8 \pm 6.7 vs. $16.5 \pm 6.6 \%, p<0.01)$. In conclusion, joint symbolic dynamics provides an efficient technique for the analysis of cardio-respiratory interaction.
\end{abstract}

\section{INTRODUCTION}

The association between heart rhythm and respiration, termed as cardio-respiratory interaction, has long been recognized [1], [2]. Respiratory sinus arrhythmia (RSA) is a well-known phenomenon of cardio-respiratory interaction [3], which is a strong modulatory effect of respiration on heart rate [4]. Although conventional signal processing techniques such as cross-correlation and power spectral density analysis have shown linear dependencies between heart rate and respiratory rate [5], [6], they often prove to be inadequate for characterizing the complex dynamics of biological signals being inherently non-linear, non-stationary and containing superimposed noise [7], [8]. Symbolic dynamics, a concept which provides a simplified description of the system's dynamics through transformation of the time series into a symbol sequence achieved by a coarse-graining procedure, has successfully been applied in several studies [9]-[11] and in the quantification of cardio-respiratory interaction [12], [13]. It has been suggested that data analyses using joint symbolic dynamics provide improved performance compared to time-domain techniques [13], [14].

It has previously been reported that delays in coupling between two systems can have significant influence on their dynamical behaviour [15]-[17]. In our previous study [18], we have suggested that the delay in heart rhythm in regard to the respiratory cycle should be taken into account in the study of interaction between cardiac and respiratory rhythms, as this substantially enhances the sensitivity of the detection of cardio-respiratory interaction.

A preferred approach to evaluating the effectiveness of analysis methods is to perform initial experiments with simulated data. In this paper we generated two non-linearly coupled systems using the Lorenz system and an autoregressive process and studied their interaction using the approach based on joint symbolic dynamics introduced in our previous study [13]. We then applied this technique on healthy subjects to study the effect of age on cardio-respiratory interaction. We hypothesized that cardio-respiratory interaction decreases with age.

\section{Methods}

\section{A. Joint Symbolic Dynamics}

Symbolic analysis involves transformation of time series into a series of discretized symbols to extract information about the behavior of the system generating the time series. From the vectors of the two time series $x$ and $y$, two symbolic sequences $s^{x}$ and $s^{y}$ were formed using the transformation rule below, based on the differences between successive $x$ and $y$ values, respectively,

$$
\begin{gathered}
s_{i}^{x}= \begin{cases}0 & \text { if } x_{i+1}-x_{i}<0, \\
1 & \text { if } x_{i+1}-x_{i}>0, \\
2 & \text { if } x_{i+1}-x_{i}=0\end{cases} \\
s_{i}^{y}= \begin{cases}0 & \text { if } y_{i+1}-y_{i}<0, \\
1 & \text { if } y_{i+1}-y_{i}>0, \\
2 & \text { if } y_{i+1}-y_{i}=0 .\end{cases}
\end{gathered}
$$

Using the symbol vectors $\mathrm{s}^{x}$ and $\mathrm{s}^{y}$, we constructed series of words, $w_{3}^{x}$ and $w_{3}^{y}$ of length ' 3 '-containing 3 successive 
TABLE I

TRANSFORMATION OF TIME SERIES' $x_{i}$ AND $y_{i}$ INTO SYMBOL VECTORS, $\mathrm{S}^{x}$ AND S $^{y}$, AND WORDS $w_{3 j}^{x}$ AND $w_{3}^{y}$ OF LENGTH 3, RESPECTIVELY. THE WORDS CAN BE PLACED IN A $27 \times 27$ MATRIX. IN THE TABLE, THE STAR INDICATES THAT SEQUENCE OF SYMBOLS IN $w_{3 j}^{x}$ CORRESPONDS TO $w_{3 j}^{y}$ AND HENCE IS CONSIDERED COORDINATED. THE PLUS SIGN INDICATES THE PAIR OF WORDS WITH A DIFFERENCE IN SEQUENCE OF SYMBOLS.

$x_{i}$
\begin{tabular}{|r|c|c|c|c|c|c|}
\hline 1.29 & 1.96 & 1.58 & 0.41 & 0.06 & 0.11 & 0.23 \\
\hline$s_{i}^{x}:$ & 1 & 0 & 0 & 0 & 1 & 1 \\
\hline$w_{3 j}^{x}:$ & - & - & 100 & 000 & 001 & 011
\end{tabular}

$y_{i}$
\begin{tabular}{|c|c|c|c|c|c|c|}
\hline 0.69 & 1.19 & 2.08 & 1.79 & 1.46 & 2.53 & 3.62 \\
\hline$s_{i}^{y}:$ & 1 & 1 & 0 & 0 & 1 & 1 \\
\hline$w_{3 j}^{y}:$ & - & - & 110 & 100 & 001 & 011
\end{tabular}

\begin{tabular}{|c|c|c|c|c|c|c|c|c|}
\hline \multicolumn{7}{|c|}{$w_{3 j}^{x}$} \\
\hline$w_{3 j}^{y}$ & 000 & 001 & 010 & 011 & 100 & 101 & $\ldots$ & 222 \\
\hline 000 & & & & & & & & \\
\hline 001 & & $*$ & & & & & & \\
\hline 010 & & & & & & & & \\
\hline 011 & & & & $*$ & & & & \\
\hline 100 & + & & & & & & & \\
\hline 101 & & & & & & & & \\
\hline 110 & & & & & + & & & \\
\hline$\ldots$ & & & & & & & & \\
\hline 222 & & & & & & & & \\
\hline
\end{tabular}

symbols - as it was suggested to provide more consistent results compared to results obtained using other word lengths [10], [13], [19]. Consequently, $3^{3}$ different word types were obtained for each vector.

The interaction between $x_{n}$ and $y_{n}$ was studied by comparing the $j^{\text {th }}(j=1,2, \ldots, n$, where $n$ is total number of words) word from the distributions, $w_{3 j}^{x}$ and $w_{3 j}^{y}$, of a particular word length $k$. If the sequence of symbols in $w_{3 j}^{x}$ corresponded to $w_{3 j}^{y}$ (i.e. $w_{3 j}^{x}=w_{3 j}^{y}$ ), the two words and hence the systems were considered to be coordinated. The word types span over a $27 \times 27$ vector matrix from $[000,000]^{\mathrm{T}}$ to $[222,222]^{\mathrm{T}}$ (Table I). The percentage of interaction was calculated by dividing the total count of coordinated words by the total number of words.

For the analysis of cardio-respiratory interaction, we replaced the ' $x$ ' and ' $y$ ' terms in equations 1 and 2 with $\mathrm{R}$ $R$ interval ' $R R$ ' and respiratory phases 'RP', as described previously [13].

\section{B. Analysis of joint symbolic dynamics approach using Lorenz system and an autoregressive model}

We used the Lorenz system to generate one of our non-linear dynamic system. The Lorenz system is defined as a threedimensional dynamical system, represented by three variables, $x, y$ and $z$, that exhibits chaotic behavior. The equations, introduced by Lorenz [20], that govern the Lorenz system are given as
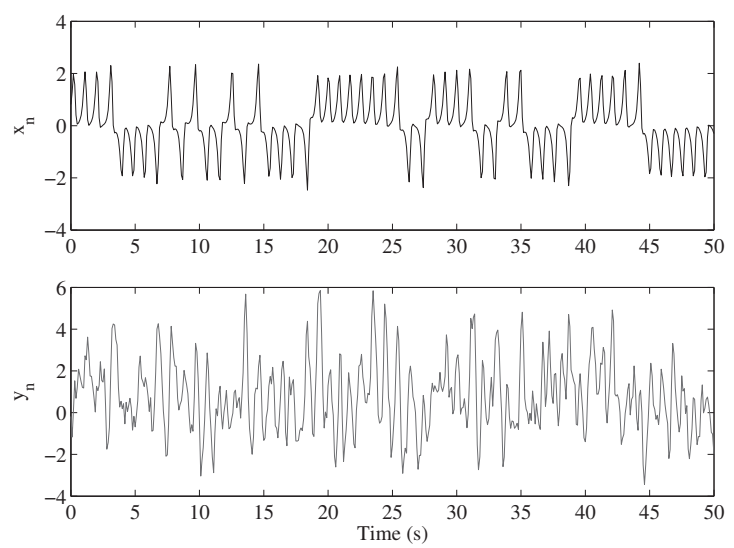

Fig. 1. Figure representing the time series of nonlinear system, $y_{n}$, coupled with the $x$-component of the Lorenz system, $x_{n}$.

$$
\begin{aligned}
& \frac{d x}{d t}=\sigma(y-x), \\
& \frac{d y}{d t}=x(\rho-z)-y, \\
& \frac{d z}{d t}=x y-\beta z .
\end{aligned}
$$

where $\sigma$ and $\rho$ are the Prandtl and Rayleigh numbers.

By solving the three differential equations, the three components of the Lorenz-system, $x, y$ and $z$ are obtained. For our analysis we considered the $x$-component of the Lorenz system. A second order auto-regressive process was used to generate the time series of the second non-linear system $y_{n}$, coupled with $x_{n}$, given by

$$
y_{n}=1.095 y_{n-1}-0.4000 y_{n-2}+0.700 \xi_{n}+0.300 x_{n}^{2} \text {. }
$$

where $\xi$ is white noise and $x$ is normalized to its standard deviation.

The time series of the two coupled systems $x_{n}$ and $y_{n}$ are shown in figure 1 . Initially, we calculated the probability of appearance of similar words, $P$, at a particular instant in both the series, by dividing the total number of words in a series $\left(n_{x}\right)$ or $\left(n_{y}\right)$ by the total combination of words from both the series, given by

$$
P=\frac{n_{x} \text { or } n_{y}}{n_{x} \times n_{y}} .
$$

Consequently, joint symbolic dynamics was applied on the coupled systems to determine the percentage of interaction between the two systems. In order to determine whether joint symbolic dynamics is an effective approach for detecting interaction between non-linear systems, we randomized the data by shuffling the values of $x_{n} 50$ times. For the purpose of statistical analysis, and considering that the values of white noise change in every realization, each process was iterated 20 times to generate a sequence of results. 


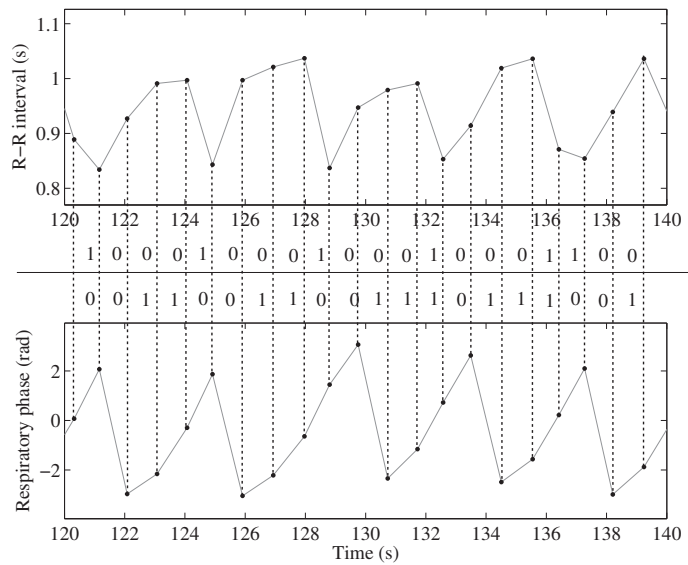

Fig. 2. Transformation of R-R intervals and R-instant respiratory phases into symbol vectors. The top panel shows the R-R intervals as obtained from the time points of R-peaks while the bottom panel represents the respiratory phase cycle and the respiratory phases at the time points of R-peaks (dots and dotted lines represent the time points and the correspondence between the two time series, respectively). The symbolic sequences obtained from the corresponding time series are shown below and above the top and bottom panels, respectively.

\section{Subjects}

ECG and respiratory signals of two groups of healthy subjects, 20 young (range: 21-34 yrs, mean: $27 \mathrm{yrs}$ ) and 20 elderly (range: 68-85 yrs, mean: 74 yrs) from the Physionet database were collected for this study. Each group consisted of 10 men and 10 women. Subjects were non-smokers and had no medical condition/history.

\section{Data Analysis - ECG and respiration}

The sampling rate of ECG and respiratory signals were 250 $\mathrm{Hz}$ and the recording time was 120 minutes in the supine position for each subject. Custom written computer software developed under MATLAB ${ }^{\circledR}$ was used to detect the R-peaks from the recorded ECG signal using parabolic fitting, where a parabola of the length based on the sampling frequency is fitted around the $\mathrm{R}$-wave to determine the $\mathrm{R}$-wave maximum. The R-R time series were visually scanned for artifacts.

To remove noise, respiratory signals were low-pass filtered at $1.0 \mathrm{~Hz}$ using a zero-phase forward and reverse digital filter, which first filtered the raw signal in the forward direction using a $4^{\text {th }}$ order Butterworth filter, and subsequently filtered the reversed signal. The resultant signal had zero phase distortion. The phases of the filtered respiratory signal were calculated using Hilbert transform.

From the vectors of the R-R time series and the series of respiratory phases at the instants of R-peaks, we established two symbolic sequences, as shown in figure 2 , based on the differences between successive R-R intervals and R-instant respiratory phases, respectively [13].

\section{E. Calculation of time delays for enhanced detection of} cardio-respiratory interaction

According to our previous studies, there exists a delay in heart rhythm in regard to the respiratory cycle which should be

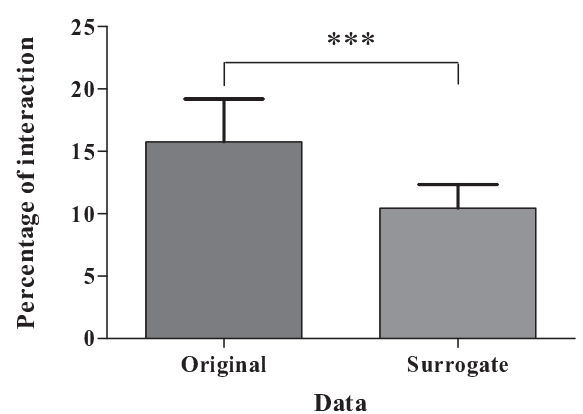

Fig. 3. Interaction between two coupled systems using original and surrogate data. A significant decrease in interaction was observed using surrogate data as compared to original coupled data. $* * *$ represents $p<0.0001$.

taken into account during the study of coordination between cardiac and respiratory rhythms [13], [18]. The most common and widely used tool for the calculation of time delay is the cross-correlation function. The linear association between $\mathrm{R}-$ $\mathrm{R}$ intervals, $\mathrm{RR}$, and the respiratory phases, $\phi_{r}(k)$, can be assessed by determining the Pearson correlation coefficients for the correlation between $\mathrm{RR}$ and $\cos \phi_{r}(k), r_{R C}=\mathrm{c}(\mathrm{RR}(i)$, $\left.\cos \phi_{r}\left(k_{i}\right)\right), \mathrm{RR}$ and $\sin \phi_{r}(k), r_{R S}=\mathrm{c}\left(\mathrm{RR}(i), \sin \phi_{r}\left(k_{i}\right)\right)$, and $\cos \phi_{r}(k)$ and $\sin \phi_{r}(k), r_{C S}=\mathrm{c}\left(\cos \phi_{r}\left(k_{i}\right)\right.$, sin $\left.\phi_{r}\left(k_{i}\right)\right)$, and subsequently determining the angular-linear correlation coefficient, $r_{R P}$, defined as [21]

$$
r_{R P}=\sqrt{\frac{r_{R C}^{2}+r_{R S}^{2}-2 r_{R C} r_{R S} r_{C S}}{1-r_{C S}^{2}}} .
$$

To introduce a delay of $\tau$ R-peaks, the parameters $r_{R C}, r_{R S}$ and $r_{C S}$ were substituted with $r^{\prime}{ }_{R C}=\mathrm{c}\left(\mathrm{RR}(i+\tau), \cos \phi_{r}\left(k_{i}\right)\right)$, $r^{\prime}{ }_{R S}=\mathrm{c}\left(\mathrm{RR}(i+\tau), \sin \phi_{r}\left(k_{i}\right)\right), r^{\prime}{ }_{C S}=\mathrm{c}\left(\cos \phi_{r}\left(k_{i}\right), \sin \phi_{r}\left(k_{i}\right)\right)$, where $\tau=-6,-5, \ldots, 0, \ldots,+5,+6$ beats. Circular statistics analysis was performed using the CircStat MATLAB ${ }^{\circledR}$ toolbox and codes described in [22]. For our analysis we selected the $\tau$ delayed RR time series that provided the highest correlation between the delayed R-R intervals and respiratory phases.

\section{F. Statistical analysis}

GraphPad Prism version 5.01 for Windows (GraphPad Software, San Diego California USA, www.graphpad.com) was used for statistical analysis. Considering normally distributed data, we investigated the interaction between nonlinear systems using coupled and surrogate data and also cardio-respiratory interaction between two age groups using Student's t-test. Values with $p<0.05$ were considered statistically significant. Data were expressed as mean \pm standard deviation (SD).

\section{RESULTS}

\section{A. Theoretical Probability Analysis}

Since 3 symbols $(0,1$ and 2$)$ were used to form words of length 3 for the analysis of interaction between systems, the probability of appearance of one word out of total possible words in a series is $\frac{1}{3^{3}}=0.037$. Consequently, the probability 


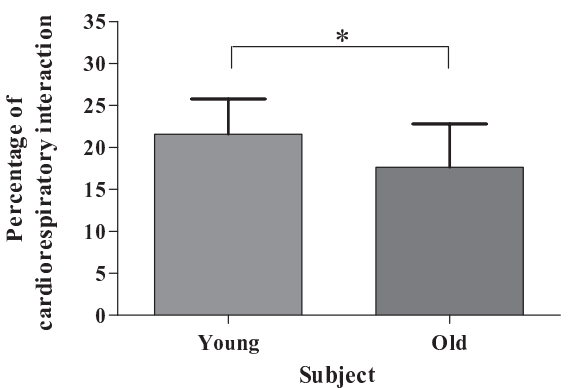

Fig. 4. Age effect on cardio-respiratory interaction using original data. Elderly subjects showed a lower percentage of interaction compared to young subjects. * represents $p<0.05$.

of appearance of similar words in both the series at a particular instant would be $\frac{3^{3}}{3^{3} \times 3^{3}}=3.7 \%$. However, we observed that although symbol ' 2 ', representing no difference between two successive values in a series, was assigned in the formula, it rarely appeared in the symbolic sequences. Based on this observation the probability was recalculated to be $\frac{2^{3}}{2^{3} \times 2^{3}}=12.5 \%$.

\section{B. Analysis of joint symbolic dynamics of the non-linearly coupled system}

Using the joint symbolic dynamics approach for the coupled systems defined by equations 3 and 4, an overall interaction of $15.8 \pm 3.4 \%$ was observed for 20 realizations (figure 3). After shuffling the values of $x_{n} 50$ times and calculating its interaction with the original $y_{n}$ for 20 realizations using joint symbolic dynamics approach, a significant decrease in the percentage of interaction was observed (15.8 \pm 3.4 vs. $10.5 \pm 1.9 \%, p<0.0001)$, see figure 3 .

\section{Age effect on cardio-respiratory interaction}

The interaction between cardiac and respiratory cycles significantly decreased in elderly subjects as compared to young subjects ( $17.6 \pm 5.1$ vs. $21.6 \pm 4.2 \%, p<0.05$, respectively), see figure 4. After choosing the delayed R-R interval that provided the highest correlation with the respiratory phases, cardiorespiratory interaction showed a relative and more significant decrease in elderly subjects as compared to young subjects ( $16.5 \pm 6.6$ vs. $22.8 \pm 6.7 \%, p<0.01$, respectively), see figure 5 .

\section{Discussion}

In this paper we used the Lorenz system and an autoregressive process to generate two coupled systems for the analysis and experimental validation of the approach based on joint symbolic dynamics, designed for the detection of cardiorespiratory interaction. Using this method in healthy subjects, a significant decrease in cardio-respiratory interaction was observed in elderly subjects as compared to young subjects.

In a study by Caminal et al. [14], the analysis of respiratory data using symbolic dynamics has been suggested to provide better results than time-domain analyses, but the transformation of respiratory time series into symbolic sequences involved different parameters whose values required to be

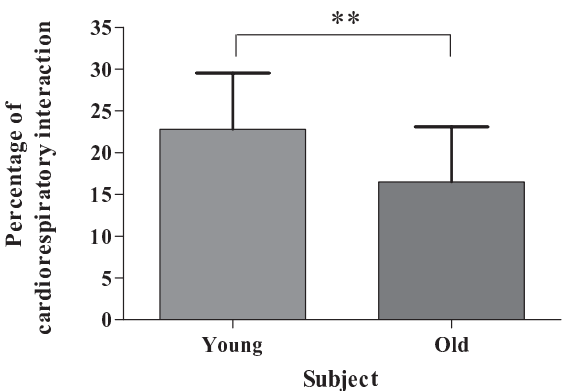

Fig. 5. Age effect on cardio-respiratory interaction using delayed R-R intervals. By appropriate delaying of the R-R time series, an increase in the overall percentage of interaction in young subjects and more significant difference in cardio-respiratory interaction between young and elderly subjects was observed. $* *$ represents $p<0.01$.

suitably selected. Our recent study introduced a new method based on joint symbolic dynamics for the investigation of cardio-respiratory interaction that involves only two parameters and is based on the changes in consecutive respiratory phases corresponding to the changes in R-R intervals [13]. The approach based on joint symbolic dynamics has also been suggested to provide improved performance in detecting changes in cardio-respiratory interactions [13]. However, to validate the effectiveness of the proposed approach, it is important to use simulated data [23]. In addition, cross-correlation and coherence functions, which determine the presence of a particular functional relationship between two time series, are based on linear measures and hence can detect little or no couplings between non-linear systems. In order to further evaluate the sensitivity and effectiveness of joint symbolic dynamics in detecting coupling between non-linear systems, we used surrogate data for our analysis.

For our simulated data analysis, we initially determined the probability of appearance of similar words in both the time series. For words of length ' 3 ' and using ' 3 ' different symbols, the probability of interaction appeared to be very low $(3.7 \%)$. However, two successive values in either of the time series of simulated data were hardly similar and hence one of the defined symbols in each of the two series remained unused. Based on this observation, a probability of interaction of $12.5 \%$ was estimated. Using shuffled values (surrogate) of $x_{n}$, and hence affecting the coupling, an interaction of $10.5 \%$ with $y_{n}$ was obtained, which was close to the estimated probability. Consequently, as hypothesized, a higher percentage of interaction (15.8\%) was observed when the systems were coupled. This would suggest that the joint symbolic dynamics approach can effectively detect interaction between two nonlinear coupled systems.

Respiratory sinus arrhythmia (RSA), modulation of heart rate by respiration, is influenced by age and is more prominent in children than in adults [24], [25]. Our analysis based on joint symbolic dynamics, which resembles the definition of RSA, showed a significant decrease in cardio-respiratory interaction in elderly subjects compared to young subjects. Also, more 
significant differences were observed after introducing a time delay between cardiac and respiratory symbol strings based on the highest correlation between R-R intervals and respiratory phases, which is in line with our previous studies [13], [18].

From this study it appears that the approach based on joint symbolic dynamics provides a simple technique for the effective quantification of interaction between two non-linearly coupled systems.

\section{CONCLUSion}

Cardio-respiratory interaction in healthy subjects is influenced by age and is more prominent in young subjects as compared to elderly subjects. Joint symbolic dynamics provides a simple tool to quantity the relation between cardiac and respiratory rhythms.

\section{REFERENCES}

[1] C. Schafer, M. G. Rosenblum, J. Kurths, and H. H. Abel, "Heartbeat synchronized with ventilation," Nature, vol. 392, no. 6673, pp. 239-40, 1998.

[2] C. Schafer, M. G. Rosenblum, H. H. Abel, and J. Kurths, "Synchronization in the human cardiorespiratory system," Phys Rev E Stat Phys Plasmas Fluids Relat Interdiscip Topics, vol. 60, no. 1, pp. 857-70, 1999.

[3] G. G. Berntson, J. T. Cacioppo, and K. S. Quigley, "Respiratory sinus arrhythmia: Autonomic origins, physiological mechanisms, and psychophysiological implications," Psychophysiology, vol. 30, no. 2, pp. 183-196, 1993.

[4] L. Bernardi, C. Porta, A. Gabutti, L. Spicuzza, and P. Sleight, "Modulatory effects of respiration," Autonomic Neuroscience: Basic \& Clinical, vol. 90, no. 1, pp. 47-56, 2001.

[5] G. Baselli, S. Cerutti, S. Civardi, D. Liberati, F. Lombardi, A. Malliani, and M. Pagani, "Spectral and cross-spectral analysis of heart rate and arterial blood pressure variability signals," Comput Biomed Res, vol. 19, no. 6 , pp. $520-34,1986$.

[6] S. Akselrod, S. Eliash, O. Oz, and S. Cohen, "Hemodynamic regulation in shr: investigation by spectral analysis," American Journal of Physiology - Heart and Circulatory Physiology, vol. 253, no. 1, pp. H176-H183, 1987.

[7] D. Hoyer, D. Kaplan, F. Schaaff, and M. Eiselt, "Determinism in bivariate cardiorespiratory phase-space sets," IEEE Eng Med Biol Mag, vol. 17 , no. 6 , pp. $26-31,1998$.

[8] M. G. Rosenblum, J. Kurths, A. Pikovsky, C. Schafer, P. Tass, and H. H. Abel, "Synchronization in noisy systems and cardiorespiratory interaction," IEEE Eng Med Biol Mag, vol. 17, no. 6, pp. 46-53, 1998.

[9] A. Voss, J. Kurths, H. Kleiner, A. Witt, N. Wessel, P. Saparin, K. Osterziel, R. Schurath, and R. Dietz, "The application of methods of nonlinear dynamics for the improved and predictive recognition of patients threatened by sudden cardiac death," Cardiovascular Research, vol. 31, no. 3, pp. 419-433, 1996.
[10] M. Baumert, T. Walther, J. Hopfe, H. Stepan, R. Faber, and A. Voss, "Joint symbolic dynamic analysis of beat-to-beat interactions of heart rate and systolic blood pressure in normal pregnancy," Medical and Biological Engineering and Computing, vol. 40, no. 2, pp. 241-245, 2002.

[11] M. Baumert, V. Baier, S. Truebner, A. Schirdewan, and A. Voss, "Short- and long-term joint symbolic dynamics of heart rate and blood pressure in dilated cardiomyopathy," IEEE Transactions on Biomedical Engineering, vol. 52, no. 12, pp. 2112-2115, 2005.

[12] M. Schiek, F. R. Drepper, R. Engbert, H. H. Abel, and K. Suder, Nonlinear analysis of physiological data. Springer, 1998, ch. Cardiorespiratory synchronization, pp. 191-209.

[13] M. Kabir, D. Saint, E. Nalivaiko, D. Abbott, A. Voss, and M. Baumert, "Quantification of cardiorespiratory interactions based on joint symbolic dynamics," Annals of Biomedical Engineering, vol. 39, no. 10, pp. 2604 2614, 2011, doi: 10.1007/s10439-011-0332-3.

[14] P. Caminal, M. Vallverdu, B. Giraldo, S. Benito, G. Vazquez, and A. Voss, "Optimized symbolic dynamics approach for the analysis of the respiratory pattern," IEEE Transactions on Biomedical Engineering, vol. 52, no. 11, pp. 1832-1839, 2005.

[15] E. Niebur, H. G. Schuster, and D. M. Kammen, "Collective frequencies and metastability in networks of limit-cycle oscillators with time delay," Physical Review Letters, vol. 67, no. 20, pp. 2753-2756, 1991.

[16] M. K. S. Yeung and S. H. Strogatz, "Time delay in the kuramoto model of coupled oscillators," Physical Review Letters, vol. 82, no. 3, pp. 648651, 1999.

[17] D. V. Ramana Reddy, A. Sen, and G. L. Johnston, "Experimental evidence of time-delay-induced death in coupled limit-cycle oscillators," Physical Review Letters, vol. 85, no. 16, pp. 3381-3384, 2000.

[18] M. Kabir, D. Saint, E. Nalivaiko, D. Abbott, and M. Baumert, "Time delay correction of the synchrogram for optimized detection of cardiorespiratory coordination," Medical \& Biological Engineering \& Computing, 2011, doi: 10.1007/s11517-011-0822-3.

[19] P. Caminal, B. Giraldo, M. Vallverd, S. Benito, R. Schroeder, and A. Voss, "Symbolic dynamic analysis of relations between cardiac and breathing cycles in patients on weaning trials," Annals of Biomedical Engineering, vol. 38, no. 8, pp. 2542-2552, 2010.

[20] E. N. Lorenz, "Deterministic nonperiodic flow." Journal of the Atmospheric Sciences, vol. 20, pp. 130-141, 1963.

[21] J. H. Zar, "Circular distributions: Hypothesis testing," in Biostatistical analysis, 4th ed., T. Ryu, Ed. New Jersey: Prentice-Hall, 1999, pp. 616-663, 570.15195Z36b.4.

[22] P. Berens, "Circstat: A matlab toolbox for circular statistics," Journal of Statistical Software, vol. 31, no. 10, pp. 1-21, 2009.

[23] A. Pikovsky, M. Rosenblum, and J. Kurths, Synchronization: A universal concept in nonlinear sciences. Cambridge University Press, 2001, ch. Synchronization of chaotic systems, pp. 137-152.

[24] W. Hrushesky, D. Fader, O. Schmitt, and V. Gilbertsen, "The respiratory sinus arrhythmia: a measure of cardiac age," Science, vol. 224, no. 4652, pp. 1001-1004, 1984.

[25] F. Yasuma and J.-i. Hayano, "Respiratory sinus arrhythmia: why does the heartbeat synchronize with respiratory rhythm?" Chest, vol. 125, no. 2, pp. 683-690, 2004. 